\title{
Obstetrics ICU admissions: challenges faced at a tertiary referral centre
}

\author{
Shehla Jamal, Archana Mehta*, Neerja Goel, Mayuri Ahuja, Naima Afreen, Sweety Malik
}

Department of Obstetrics and Gynecology, SMS and R, Sharda University, Greater Noida, Uttar Pradesh, India

Received: 02 March 2018

Accepted: 28 March 2018

\section{*Correspondence:}

Dr. Archana Mehta,

E-mail: archiedrmehta@gmail.com

Copyright: (C) the author(s), publisher and licensee Medip Academy. This is an open-access article distributed under the terms of the Creative Commons Attribution Non-Commercial License, which permits unrestricted non-commercial use, distribution, and reproduction in any medium, provided the original work is properly cited.

\section{ABSTRACT}

Background: Management of critically ill patients in obstetrics is a challenge owing to the changed physiology and unpredictability of the disease behaviour. Stratification strategy for early admission to obstetric ICU is imperative to reduce maternal morbidity and mortality. Due to lack of formal surveys regarding obstetrical ICU in our country, there is lack of precise national data on obstetrical ICU mortality. Aim of the present study was to estimate the obstetric ICU admission rate, to study the pattern of causative aetiology and to study the complications developed in ICU.

Methods: The present study is a retrospective analytical study done in the Department of Obstetrics and Gynecology, from January 2015 to August 2017.

Results: During the study period there were a total of 8466 obstetrical admissions, 2508 deliveries and 104 ICU admissions. Out of 104, analysis was done on 71 patients. Hypertensive disorders of the pregnancy were associated with maximum number of admissions (47.8\%), followed by postpartum haemorrhage (12.6\%). Maximum admissions were in postpartum period (63.4\%). Anaemia was associated with $35.2 \%$ of the cases and it was closely followed by sepsis $(28.2 \%)$. Mechanical ventilation was required for $30.9 \%$ of the patients.

Conclusions: Hypertensive disorders of the pregnancy, haemorrhage and sepsis are the major risk factors for ICU admission in obstetric population.

Keywords: Hypertensive disorders of pregnancy, Obstetrical ICU

\section{INTRODUCTION}

Management of critically ill patients in obstetrics is a challenge owing to the changed physiology and unpredictability of the disease behaviour. Admission of obstetric patients occurs at approximately $0.1-0.9 \%$ of the total deliveries. ${ }^{1}$ Overall maternal death rate in the ICU varies from $3.4-21 \% .^{2}$ Delay in diagnosing the illness and infrequent admission of the obstetric patients to ICU, results in high morbidity and mortality. Intensive care is appropriate for patients in need of advanced respiratory support and who require support of two or more organ systems. In patients with chronic impairment of one or more organ systems who also have superadded acute failure of other organs, ICU care has shown to improve prognosis. Early referral to ICU is extremely important. If referral is delayed until the patient's life is clearly at risk, the chances of full recovery are jeopardised. Stratification strategy for early admission to obstetric ICU is imperative to reduce maternal morbidity and mortality. Studies done from different parts of India have demonstrated different rates of utilisation of ICU for imparting care. ${ }^{3}$ Analysis of obstetric ICU admission gives an insight into the pattern of the attributable causes of critical illness prevalent in obstetric population of that area. ICU utilisation serves as an objective marker for the quality of care in that area. Such an effort is equally important for early identification of end stage organ failure to ensure appropriate intervention

High income countries have shown a consistent decline in maternal mortality over the past six decades, but low 
income countries have failed to demonstrate similar trends. According to a large systematic review, ICU maternal mortality is $8-40 \%$ in low income countries as compared to $1-3.4 \%$ in high income countries. ${ }^{2}$

Due to lack of formal surveys regarding obstetrical ICU in our country, there is lack of precise national data on obstetrical ICU mortality. Small sample studies from various public health hospitals have revealed that $0.4-4 \%$ of obstetric admissions are in ICU. The rate of admission to ICU is an important predictor of maternal mortality and has been an important criterion for evaluation of subacute maternal morbidity evaluation. Hence, the present survey was undertaken to evaluate the causes, outcomes and course of events of obstetrical patients in ICU at our centre.

Objectives of present study were to estimate the obstetric ICU admission rate, to study the pattern of causative aetiology and to study the complications developed in ICU.

\section{METHODS}

The present study is a retrospective analytical study done in the department of obstetrics and gynaecology, from January 2015 to August 2017. All the obstetric cases and upto six weeks postpartum, admitted to ICU (directly/transferred), were included in the study. Patient case sheets having incomplete information, and the patients leaving hospital against medical advice were excluded out from the analysis. The records were procured from the medical records department of the hospital.

Hypertensive disorders of pregnancy included severe preeclampsia and eclampsia. Severe anaemia in pregnancy or postpartum was defined as haemoglobin $<7 \mathrm{gm} \%$. Post-partum haemorrhage was defined as acute blood loss causing haemodynamic instability or need of blood transfusion. Ante partum haemorrhage was defined as bleeding from the genital tract after 28 weeks till before labor.

Acute respiratory distress was defined as tachypnoea with respiratory rate $>30$ per minute, or bradypnoea with respiratory rate $<6$ per minute, cyanosis, oxygen saturation $<90 \%$ for more than 10 minutes. Sepsis was defined as TLC $>12,000 / \mathrm{mm} 3$, or less than $4,000 / \mathrm{mm} 3$. Hepatic dysfunction was defined as serum bilirubin $>6$ $\mathrm{mg} / \mathrm{dl}$. Cardiovascular dysfunction was termed when patient had shock, required cardiopulmonary resuscitation, required ionotropes for maintenance of vital organ perfusion and $\mathrm{pH}<7.1$ on $\mathrm{ABG}$ analysis. Coagulation failure was defined when there was failure of stable clot formation on bed side test, deranged PT/INR according to the reference laboratory parameters, thrombocytopenia $<50,000 / \mathrm{mm}^{3}$, need of transfusing $>4$ units of blood products. Renal dysfunction was described as creatinine levels $>1.2$ and oliguria not responding to fluid challenge and diuretics. Prolonged stay in ICU was stay in ICU for more than 72 hours. Events in ICU were classified as development of shock, CPR, tracheostomy and death. Prolonged NICU admission was defined as stay in NICU for $>24$ hours. Collected data was analysed for demographic profile, reason for ICU admission, stay in ICU, interventions done, mortality, blood and its products transfused and neonatal outcome. The result was analysed using percentage method.

\section{RESULTS}

During the study period there were a total of 8466 obstetrical admissions, 2508 deliveries and 104 ICU admissions. Out of 104, analysis was done on 71 patients as rest had incomplete information. Calculated ICU utilisation rate was $1.3 \%$. Majority of the patients belonged to age group of 20-34 years (mean age $24 \pm 2$ ), multiparous with mean birth order 2 , and belonged to rural areas (Table 1).

Table 1: Demographic and maternal characteristics.

\begin{tabular}{|lll|}
\hline Characteristics & Number (n) & Percentage (\%) \\
\hline Age (years) & & \\
\hline$<19$ & 6 & 8.4 \\
\hline $20-34$ & 61 & 85.9 \\
\hline$>35$ & 4 & 5.7 \\
\hline Parity & \\
\hline Primi & 28 & 39.4 \\
\hline Multi & 43 & 60.6 \\
\hline Area & & \\
\hline Rural & 58 & 81.7 \\
\hline Urban & 13 & 18.3 \\
\hline Gestation (weeks) & & \\
\hline$<28$ & 7 & 9.9 \\
\hline $28-42$ & 45 & 26.7 \\
\hline Postpartum & 19 & 63.4 \\
\hline
\end{tabular}

Maximum admissions were in postpartum period $(63.4 \%)$. We observed a caesarean delivery incidence of $50.7 \%$ in the patients admitted to ICU and a still birth rate of $35.6 \%$ was observed (Table 2).

Table 2: Maternal and fetal outcome.

\begin{tabular}{|lll|}
\hline & Number (n) & Percentage (\%) \\
\hline Vaginal delivery & 9 & 12.6 \\
\hline Caesarean & 36 & 50.7 \\
\hline Laparotomy & 10 & 14.1 \\
\hline Others & 16 & 22.6 \\
\hline Neonatal & & \\
\hline IUFD & 16 & 35.6 \\
\hline Live birth & 29 & 64.4 \\
\hline
\end{tabular}

Hypertensive disorders of the pregnancy were associated with maximum number of admissions (47.8\%), followed by postpartum haemorrhage $(12.6 \%)$ (Figure 1$)$. 


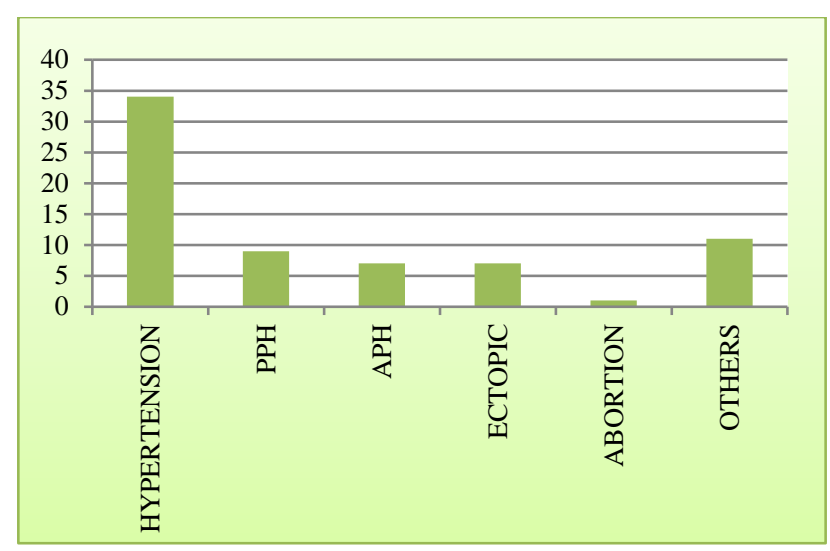

Figure 1: Distribution according to the cause of ICU admission.

When analysis was done on the basis of organ system involved, it was observed that anaemia was associated with $35.2 \%$ of the cases and it was closely followed by sepsis $(28.2 \%)$. Around $15.5 \%$ had coagulopathy (DIC) (Figure 2).

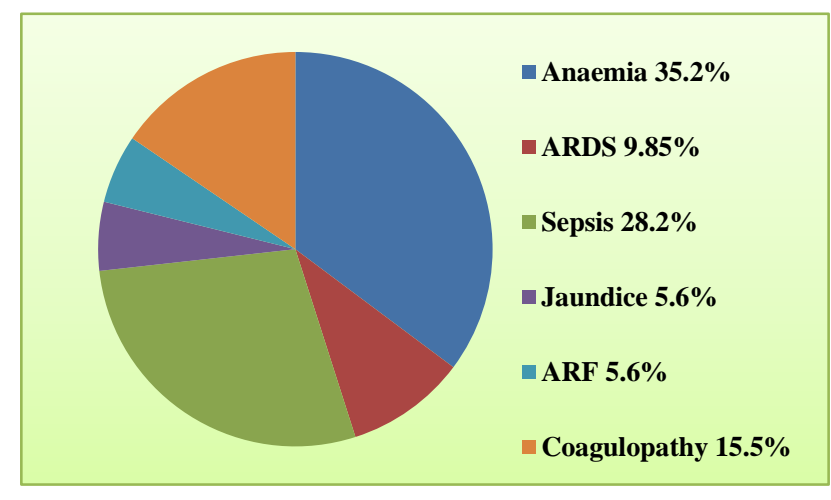

Figure 2: Distribution according to the system involved.

Mechanical ventilation was required for $30.9 \%$ of the patients, while tracheostomy was had to be performed in $4.2 \%$ patients (Table 3 ).

Table 3: Complications and interventions in ICU.

\begin{tabular}{|lll|}
\hline Complication & Number (n) & Percentage (\%) \\
\hline Ventilation & 22 & 30.9 \\
\hline Tracheostomy & 3 & 4.2 \\
\hline Ionotropes & 24 & 33.8 \\
\hline Sepsis & 8 & 11.3 \\
\hline High antibiotic & 20 & 28.2 \\
\hline Death & 3 & 4.2 \\
\hline
\end{tabular}

There was a significant proportion of the patients receiving blood and its products. Prolonged ICU admission was seen in $23.9 \%$ cases. While staying in ICU, $11 \%$ patients developed complications in the form of electrolyte disturbance and sepsis. There was a total of three maternal deaths in ICU in the study period. Third generation cephalosporin was the commonest used antibiotic, and $28.2 \%$ patients required higher group of antibiotics.

\section{DISCUSSION}

The present appraisal was undertaken with an aim to evaluate incidence of ICU admission at our centre, which being a tertiary care set up, caters to the referred patients from adjoining rural and urban areas. The observed rate of ICU admissions is $1.3 \%$, which is higher than the rates documented by studies done by various authors in India. ${ }^{3}$ Higher rates observed in our study might be due to the fact that our hospital has 24 hours ICU facility with fully functional blood bank and we get critical care referral from adjoining government as well as private health care set up.

Multiparity was observed as a risk factor for higher rates of admission in ICU. Higher birth order is an independent risk factor for pregnancy and puerperium related complications. ${ }^{4}$ A higher rate of ectopic, PPH was observed in multiparous patients in the present study. A total of $43 \%$ patients were referred from adjoining health care setups, and maximum admissions were made in postpartum period. Our observation is similar to the observation made by Ashraf $\mathrm{N}$ et al and Zwart JJ et al. ${ }^{5,6}$ they have also reported higher rates of admission in postpartum period.

Commonest indication for ICU admission in the present survey was hypertension (47.8\%), followed by PPH and APH. Identical findings have been made by various authors across India. ${ }^{7,8}$ This highlights hypertension as a major cause of concern which needs to be tackled at an urgent level. Major issue associated with HDP and haemorrhage in our country is the delay in referral and limited accessibility to intensive care facility. Niyazi and co-authors have also highlighted this observation. ${ }^{5}$ Addressing the requirement for easy accessibility and comprehensiveness of obstetric care will make a lasting impact towards overall reduction in morbidity and mortality. When associated comorbidities were studied, sepsis was diagnosed in $28.2 \%$ of the patients. The incidence observed is higher than the observations reported by Tripathi (16\%) and Karnad (6\%). ${ }^{9,10}$ But incidence reported by Gombar $\mathrm{S}$ et al $(27.15 \%)$ was similar to our observation. ${ }^{7}$ The differences obtained might be explained owing to the different inclusion protocols. Nonetheless, sepsis remains a largely preventable and under reported cause of maternal morbidity and mortality, not only in India, but globally also. ${ }^{11}$ Hence studies extracting such data should be encouraged to quantify the magnitude of the problem and taking measures to avert the same.

In the current study, a total of $4.2 \%$ of maternal mortality rate was observed amongst ICU admission. The observed rate is rather very low in comparison to most of the studies. ${ }^{5,12,13}$ Major cause of mortality in our patients was haemorrhage and majority presented in decompensated 
state, thus reflecting the lack of well-equipped maternal care. Authors observed a higher caesarean delivery rate $(50.7 \%)$ in the ICU cohort. Commonest indication was eclampsia $(78.1 \%)$. Need for emergent delivery in cases of severe preeclampsia and eclampsia has given rise to increased operative interference in this group of patients. The observed rate of still birth was also quite high $(35.6 \%)$, and it was associated with patients having HDP. Studies pertaining to fetal neonatal morbidities are scarce in number, but few studies have concluded a higher rate of still birth in ICU admitted patients.

It was observed that haemodynamic and respiratory complications needing ventilatory support $(30.9 \%)$ and ionotropes $(33.8 \%)$, were the commonest interventions done in ICU, and our findings corroborate well with those of Kilpatrick SJ. ${ }^{1,13}$ Higher group of antibiotics were started in $28.2 \%$ of the obstetric cohort in ICU during the study period.

The present study was limited by its retrospective design, so no inference regarding difficulties in referral process and factors inhibiting access to the best care, could be drawn. Hence, more prospective studies should be undertaken in-order to understand the real etiological pattern.

\section{CONCLUSION}

Based on the observations made, we conclude the following points:

- Hypertensive disorders of the pregnancy, haemorrhage and sepsis are the major risk factors for ICU admission during the study period.

- Ventilator and ionotropic support were the commonest interventions in ICU, although this was not correlated with the prognosis, as observed mortality rate was low in our study.

- Anaemia was a significant associated comorbidity, which fails to decrease despite exhaustive efforts.

- Audit on obstetric ICU admission should be encouraged in India in-order to validate the observations made in the present study. Such an insight will also ensure improved patient care and mark the grey areas in the present health care delivery system.

Funding: No funding sources

Conflict of interest: None declared

Ethical approval: Not required

\section{REFERENCES}

1. Kilpatrick SJ, Matthay M. Obstetric patients requiring critical care. A five-year review. Chest. 1992;101:1407-12.
2. Gilbert TT, Smulian JC, Martin AA, Ananth CV, Scorza W, Scardella AT, et al. Obstetric admissions to the intensive care unit: Outcomes and severity of illness. Obstet Gynecol. 2003;102:897-903.

3. Poornima B, Bhat R. Evaluation of obstetric admissions to intensive care unit of a tertiary referral center in coastal India. Indian J Crit Care Med. 2013 Jan-Feb;17(1):34-7.

4. Alsammani MA, Ahmed SR. Grand Multiparity. Risk factors and outcome in a tertiary hospital: a comparative study. Mater Sociomed. 2015 Aug;27(4):244-7.

5. Ashraf N, Mishra SK, Kundra P, Veena P, Soundaraghavan S, Habeebullah S. Obstetric patients requiring intensive care: a one year retrospective study in a tertiary care institute in India. Anesthesiol Res Pract. 2014;2014.

6. Zwart JJ, Dupuis JR, Richters A, Öry F, van Roosmalen J. Obstetric intensive care unit admission: a 2-year nationwide population-based cohort study. Intens Care Med. 2010 Feb 1;36(2):256-63.

7. Gombar S, Ahuja V, Jafra A. A retrospective analysis of obstetric patient's outcome in intensive care unit of a tertiary care center. J Anaesthesiol Clini Pharmacol. 2014 Oct;30(4):502.

8. Gupta S, Naithani U, Doshi V, Bhargava V, Vijay BS. Obstetric critical care: A prospective analysis of clinical characteristics, predictability, and fetomaternal outcome in a new dedicated obstetric intensive care unit. Indian J Anaesth. 2011 Mar;55(2):146-53.

9. Tripathy R, Rathore AM, Saran S. Intensive care for critically ill obstetric patients. Int J Obstet Gynecol. 2000;68(3):257-8

10. Karnad DR, Lapsia V, Krishnan A, Salvi VS. Prognostic factors in obstetric patients admitted to an Indian intensive care unit. Critic Care Med. 2004 Jun 1;32(6):1294-9.

11. Accost CD, Knight M. Sepsis and Maternal Mortality. Curr Opin Obstet Gynecol. 2013;25:10916

12. Ghike $S$, Asegaonakar P. Why Obstetric patients are admitted to Intesive Care Unit? A Retrospective Study. J South Asian Feder Obst Gynae. 2012;4(2):90-2.

13. Pattnaik T, Samal S, Behuria S. Obstetric admissions to the intensive care unit: a five year review. Int $\mathbf{J}$ Reprod Contracept Obstet Gynecol. 2015;4:1914-7.

Cite this article as: Jamal S, Mehta A, Goel N, Ahuja M, Afreen N, Malik S. Obstetrics ICU admissions: challenges faced at a tertiary referral centre. Int J Reprod Contracept Obstet Gynecol 2018;7:1840-3. 